\title{
Spin-orbit coupling effect in (Ga,Mn)As films: Anisotropic exchange interactions and magnetocrystalline anisotropy
}

\author{
S. Mankovsky, ${ }^{1}$ S. Polesya, ${ }^{1}$ S. Bornemann, ${ }^{1}$ J. Minár,${ }^{1}$ F. Hoffmann, ${ }^{2}$ C. H. Back, ${ }^{2}$ and H. Ebert ${ }^{1}$ \\ ${ }^{1}$ Department of Chemistry and Physical Chemistry, LMU Munich, Butenandtstrasse 11, D-81377 Munich, Germany \\ ${ }^{2}$ Department of Physics, Universität Regensburg, D-93040 Regensburg, Germany
}

(Received 30 August 2011; published 15 November 2011)

\begin{abstract}
The magnetocrystalline anisotropy (MCA) of (Ga,Mn)As films has been studied on the basis of ab initio electronic structure theory by performing magnetic torque calculations. An appreciable contribution to the in-plane uniaxial anisotropy can be attributed to an extended region adjacent to the surface. Calculations of the exchange tensor allow to ascribe a significant part to the MCA to the exchange anisotropy, caused either by a tetragonal distortion of the lattice or by the presence of the surface or interface.
\end{abstract}

DOI: 10.1103/PhysRevB.84.201201

PACS number(s): 75.50.Pp, 75.30.Gw, 75.70.-i

Diluted magnetic semiconductors (DMSs) are a class of materials having attractive properties for spintronic applications (e.g., see the review in Ref. 1). Many investigations in this field are focused on the (Ga,Mn)As DMS system with $1 \%-10 \%$ of $\mathrm{Mn}$ atoms which have promising features from a physical as well as technological point of view. The crucial role of valence states with respect to various magnetic properties of $(\mathrm{Ga}, \mathrm{Mn})$ As was discussed in the literature by many authors (e.g., Refs. 1-5, and 6). First of all, the valence-band holes are responsible for ferromagnetic (FM) order in the system mediating the exchange interaction between well-localized Mn magnetic moments. Spin-orbit coupling of the states at the top of valence band, being close to the Fermi level, leads to a rather strong cubic magnetocrystalline anisotropy (MCA) in bulk (Ga,Mn)As and to an in-plane biaxial MCA in the $(\mathrm{Ga}, \mathrm{Mn})$ As film on top of a GaAs substrate. ${ }^{1-3}$ In the latter case the spin-orbit coupling (SOC) makes the valence states close to $E_{F}$ sensitive to lattice distortions and is in that way responsible for the in-plane MCA due to compressive strains originating from the lattice mismatch between the $(\mathrm{Ga}, \mathrm{Mn}) \mathrm{As}$ film and GaAs substrate. ${ }^{7-17}$ As soon as the spin polarization of the valence bands becomes rather small, the MCA in $(\mathrm{Ga}, \mathrm{Mn}) \mathrm{As}$ is discussed in terms of anisotropic exchange interactions of the $\mathrm{Mn}$ atoms. ${ }^{2,3,7}$ The strength of the MCA depends on the hole concentration introduced by the Mn impurity atoms ${ }^{2,11,18,19}$ as well as on the variation of the equilibrium lattice parameter of (Ga,Mn)As, which increases with increasing Mn content and results thus in a larger lattice mismatch with the GaAs substrate.

Numerous experimental results evidenced a temperature-induced transition from the biaxial to the uniaxial in-plane anisotropy in (Ga,Mn)As films deposited on GaAs. ${ }^{11-18}$ In spite of different experimental conditions, the in-plane uniaxial anisotropy was observed for $(\mathrm{Ga}, \mathrm{Mn}) \mathrm{As}$ films in a thickness range from $25 \mathrm{~nm}$ (Ref. 20) to $500 \mathrm{~nm},{ }^{16}$ irrelevant with respect to the surface condition. So far, however, to the best of our knowledge, there is no consensus in the literature concerning the origin of the in-plane uniaxial anisotropy. Although in some recent theoretical works the origin of the uniaxial in-plane anisotropy is attributed to a trigonal distortion caused by a uniaxial or shear strain within the film plane,,$^{13,18,21}$ this type of distortion was not observed experimentally. Recently,
Werpachowska and Dietl ${ }^{22}$ suggested that the anisotropy mechanism in $(\mathrm{Ga}, \mathrm{Mn}) \mathrm{As}$ films originates from Dzyaloshinsky-Moriya interactions, without assuming any in-plane lattice distortion within their model. Another group of authors have found in the experiment ${ }^{23}$ the stacking

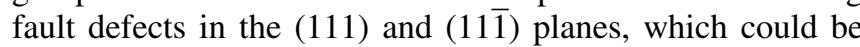
responsible for breaking the equivalence of the [110] and [11 10$]$ directions in the $(\mathrm{Ga}, \mathrm{Mn})$ As films. However, to the best of our knowledge, there is so far no experimental evidence nor theoretical description showing that these stacking faults are responsible for the in-plane uniaxial anisotropy.

In order to obtain a more detailed understanding of the subtle electronic effects which determine the MCA properties of (Ga,Mn)As films, investigations based on ab initio electronic structure calculations have been performed. Note that the films studied experimentally are too thick for performing MCA calculations that allow a direct comparison with experiments. Therefore, the present results have a semiquantitative character: The possible effects responsible for the MCA have been simulated considering tetragonally distorted bulk (Ga,Mn)As systems (to investigate the compressive straininduced in-plane anisotropy) and 8-ML (Ga,Mn)As films deposited on a GaAs substrate (to investigate the uniaxial in-plane anisotropy).

The ab initio calculations have been performed within the framework of the local spin density approximation (LSDA) of density functional theory (DFT) using the fully relativistic Korringa-Kohn-Rostoker (KKR) multiple-scattering band-structure method. ${ }^{24,25}$ For the treatment of the chemical disorder in $(\mathrm{Ga}, \mathrm{Mn})$ As alloys we applied the coherent potential approximation (CPA). Moreover, for the bulk and surface calculations we used a regular $\vec{k}$ mesh of $63 \times 63 \times 63$ points in the full three-dimensional (3D) Brillouin zone (BZ) and $63 \times 63$ points in the full two-dimensional (2D) BZ, respectively. The energy integration in torque calculations has been performed in a complex energy plane using a logarithmic energy mesh with 24 energy points. For the angular momentum expansion of the Green's function, a cutoff of $\ell_{\max }=3$ was applied.

The study of the magnetocrystalline anisotropy (MCA) was performed by calculating the magnetic torque $\vec{T}_{i}^{\left(\hat{e}_{i}\right)}=$ $-\partial E\left(\left\{\hat{e}_{k}\right\}\right) / \partial \hat{e}_{i} \times \hat{e}_{i}$ acting on the magnetic moment $\vec{m}_{i}$ of the 


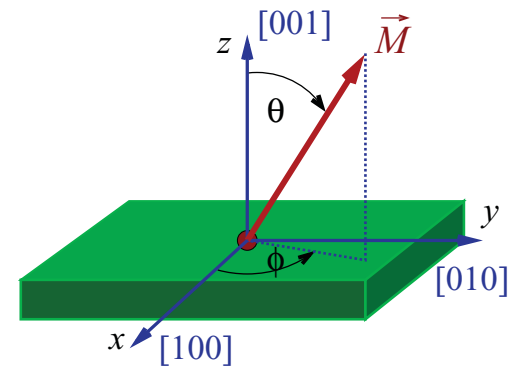

(a)

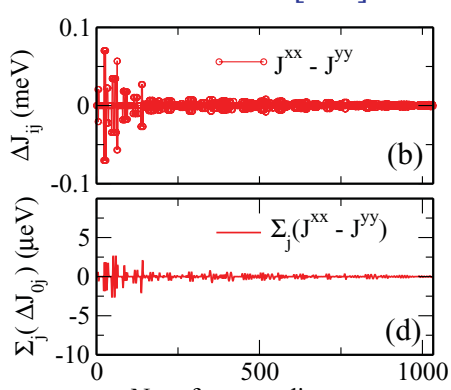

(b)

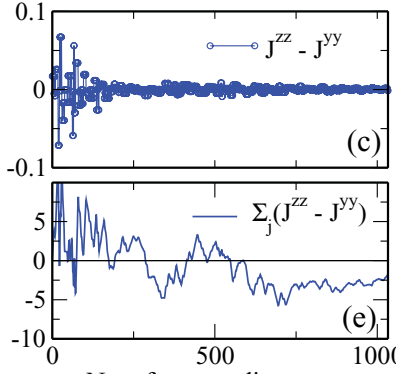

(e)
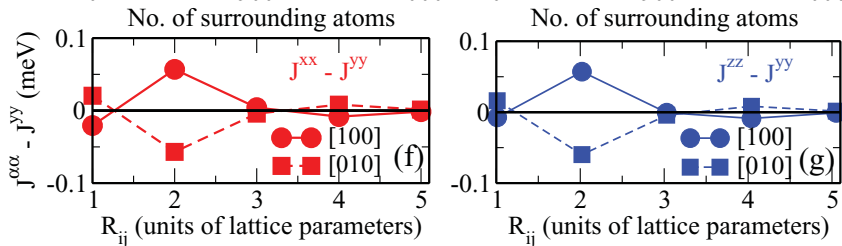

FIG. 1. (Color online) (a) Geometry for the torque calculations; $J^{x x}-J^{y y}(\mathrm{~b})$ and $J^{z z}-J^{y y}$ (c) difference for bulk (Ga,Mn)As with $5 \% \mathrm{Mn}$, with a tetragonal distortion $c / a=1.01 ; \sum_{j}\left(J_{i j}^{x x}-J_{i j}^{y y}\right)$ (d) and $\sum_{j}\left(J_{i j}^{z z}-J_{i j}^{y y}\right)$ (e) over all lattice sites up to $R_{i j} \leqslant 5 a$; comparison of $J^{x x}-J^{y y}$ (f) and $J^{z z}-J^{y y}$ (g) for $R_{i j}$ along [100] and [010] directions.

atomic site $i$, with a unit vector $\hat{e}_{i}=\vec{m}_{i} /\left|\vec{m}_{i}\right|$ pointing along the direction of the magnetization $\vec{M}$. The component of the magnetic torque with respect to the axis $\hat{u}$,

$$
T_{\hat{u}}(\theta, \phi)=-\partial E[\vec{M}(\theta, \phi)] / \partial \theta,
$$

was calculated from first-principles theory as described in Ref. 26. Here, the $\hat{u}$ vector specified by the angles $\theta$ and $\phi$ [see Fig. 1(a)] lies within the surface plane and is perpendicular to the direction of the magnetic moment $\hat{e}_{M}$. For an uniaxial anisotropy a special geometry can be used which gives a simple relationship between the magnetic torque and the energy difference between the in-plane and out-of-plane magnetization directions. Setting $\theta=\pi / 4$, the torque component $T_{\hat{u}}$ gives the $\phi$-dependent energy difference $T_{\hat{u}}(\theta=\pi / 4, \phi)=$ $E_{\|}(\phi)-E_{\perp} \cdot{ }^{26-28}$ In the case of an in-plane anisotropy these values can also be used to evaluate the anisotropy energy within the plane, comparing, in particular, the directions [110] and [110]. Below, a more detailed discussion of the origin of MCA in $(\mathrm{Ga}, \mathrm{Mn})$ As film has be done in terms of the Heisenberg model, extended to account for relativistic effects: ${ }^{28}$

$$
H=-\frac{1}{2} \sum_{i, j(i \neq j)} \hat{e}_{i} \underline{\underline{J}}_{i j} \hat{e}_{j}+\sum_{i} E_{i}^{\mathrm{MCA}}\left(\hat{e}_{i}\right) .
$$

The exchange-coupling tensor $\underline{\underline{J}}_{i j}$ was calculated as described in Ref. 29. As was shown in Refs. 28 and 30, the first term in Eq. (2) gives an additional (nonlocal) contribution to the
MCA. This yields, for example, for an easy axis parallel to the $z$ direction, the expression for the effective anisotropy coefficients $\tilde{K}_{i}=-\frac{1}{2} \sum_{j}\left(J_{i j}^{z z}-J_{i j}^{x x}\right)+K_{i}$, with $K_{i}$ being the on-site MCA coefficients. The anisotropy energy $E_{i}^{\mathrm{MCA}}\left(\hat{e}_{i}\right)$ accounts for the on-site magnetic anisotropy energy (MAE) associated with each individual moment oriented along $\hat{e}_{i}$. Assuming a collinear magnetization of the system and keeping only the terms characterizing uniaxial anisotropy, as considered here, it can be approximated in the following form: $\quad E_{i}^{\mathrm{MCA}}\left(\theta_{i}, \phi_{i}\right)=K_{\perp} \cos ^{2} \theta+K_{\| \mid} \sin ^{2} \theta \cos 2 \phi+$ $K_{\|}^{\prime} \sin ^{2} \theta \sin 2 \phi, \quad$ giving $\quad E_{\perp}=E(\theta=0)=K_{\perp} \quad$ and $E_{\|}=E(\theta=\pi / 2, \phi)=K_{||} \cos 2 \phi+K_{||}^{\prime} \sin 2 \phi$. With this, the torque component $T_{\hat{u}}=-\partial E[\vec{M}(\theta, \phi)] / \partial \theta$ corresponding to the Hamiltonian in Eq. (2) can be represented by the expression $T_{\hat{u}}(\theta, \phi)=\sin 2 \theta\left[-\frac{1}{2} \sum_{j(i \neq j)}\left(J_{i j}^{x x} \cos ^{2} \phi+\right.\right.$ $\left.\left.J_{i j}^{y y} \sin ^{2} \phi-J_{i j}^{z z}\right)+\left(K_{\|} \cos 2 \phi+K_{||}^{\prime} \sin 2 \phi-K_{\perp}\right)\right]$. For the special value $\theta=\pi / 4$ this yields the expression $E_{\|}(\phi)-E_{\perp}=\frac{1}{2} \sum_{j \neq i}\left[\left(J_{i j}^{x x} \cos ^{2} \phi+J_{i j}^{y y} \sin ^{2} \phi\right)-J^{z z}\right]+$ $\left(K_{\|} \cos 2 \phi+K_{\|}^{\prime} \sin 2 \phi-K_{\perp}\right)$, leading to the effective anisotropy constant.

In order to study the strain-induced effect in the MCA of deposited (Ga,Mn)As films, we consider at first a bulk system with tetragonal distortion (avoiding surface and interface contributions) which is then characterized by the $c / a$ ratio. Magnetic torque calculations simulating the strain-induced effects in the alloy with $5 \% \mathrm{Mn}$ yield a linear variation of the magnetic anisotropy energy $E_{[100]}-E_{[001]}$ from +3.38 to $-3.37 \mu \mathrm{eV}$ per unit cell for a $c / a$ ratio varying from 0.99 to 1.01 , i.e., the magnetic easy axis changes from an out-of-plane to an in-plane orientation, which is in line with corresponding experimental data. ${ }^{31-33}$

The magnetic dipole-dipole interactions lead in film geometry to the in-plane (uniform within the plane) anisotropy with the energy $E^{\text {dip }} \approx 0.24 \mu \mathrm{eV}$ per unit cell in the case of an alloy with $5 \% \mathrm{Mn}$ and $\mathrm{Mn}$ magnetic moments $3.7 \mu_{B}$ /atom. This is smaller by an order of magnitude than the strain-induced MCA. Since this value (shape anisotropy), which is represented per unit cell, has a rather weak dependence on the film thickness, the strain-induced MCA will have the main responsibility for the in-plane or out-of-plane direction of the magnetic anisotropy. Therefore, we will focus in the following only on the properties of the strain-induced MCA. As the [100] and [010] directions in bulk system are equivalent, this leads to the biaxial in-plane MCA with [100] and [010] being easy magnetization directions, yielding the energy difference $E_{[110]}-E_{[100]}$ below $1 \mu \mathrm{eV}$ per unit cell.

For a more detailed analysis of the relationship between the MCA and anisotropy of Mn-Mn exchange interactions, calculations of the exchange-coupling tensor elements $J_{i j}^{\alpha \beta}$ have been performed for $(\mathrm{Ga}, \mathrm{Mn}) \mathrm{As}$ with $5 \% \mathrm{Mn}$, both without any distortion as well as with a tetragonal distortion of $c / a=1.01$. For an undistorted (Ga,Mn)As system we find that the sum $\sum_{j}\left(J_{i j}^{\alpha \alpha}-J_{i j}^{\beta \beta}\right)(\alpha, \beta=x, y, z)$ over all lattice sites vanishes in the expression for the torque component $T_{\hat{u}}$. This is a consequence of the system's symmetry, in spite of the fact that the individual terms $\left(J_{i j}^{\alpha \alpha}-J_{i j}^{\beta \beta}\right)$ with $\alpha \neq \beta$ are nonzero. In the presence of a tetragonal distortion along the $z$ axis, the symmetry properties within the $x y$ plane 
[i.e., (001) plane] do not change. Therefore, summation over all lattice sites up to $R_{i j}=5 a$ (with lattice parameter $a$ ) of the $J_{i j}^{x x}-J_{i j}^{y y}$ differences [Fig. 1(b)] gives $\sum_{j}\left(J_{i j}^{x x}-J_{i j}^{y y}\right)=0$, shown in Fig. 1(c). For more details, Fig. 1(f) shows the differences $J_{i j}^{x x}-J_{i j}^{y y}$ for $\vec{R}_{i j}$ taken along the [100] and [010] directions (dashed lines). These values are finite and equal in magnitude, but they have an opposite sign and therefore cancel each other upon summation over all sites. However, due to the tetragonal distortion along $z,\left(J_{i j}^{z z}-J_{i j}^{y y}\right)$ for $\vec{R}_{i j}$ taken along the [001] and [010] directions are not equivalent [Fig. 1(f), solid lines] and thus the sum $\sum_{j}\left(J_{i j}^{z z}-J_{i j}^{y y}\right)$ over all lattice sites does not vanish anymore. The summation of $J_{i j}^{z z}-J_{i j}^{y y}$ elements [Fig. 1(c)] over all lattice sites up to $R_{i j} \leqslant 5 a$ is shown in Fig. 1(e), which gives the contribution to the uniaxial MCA that originates from the exchange anisotropy as $\approx 2.5 \mu \mathrm{eV}$. Because of the slow convergence of the sum with increasing distance, this gives only an approximation to the true contribution due to the exchange anisotropy. Nevertheless, the value obtained in this way has the same order of magnitude as the MAE obtained from our torque calculations, leading to the conclusion that the exchange anisotropy has indeed a significant impact on the total MAE.

Our present investigations of the in-plane uniaxial anisotropy have been performed for a 8-monolayer (ML)-thick (Ga,Mn)As film deposited on a semi-infinite (001)-oriented GaAs substrate. In order to distinguish the anisotropy behavior in the vicinity to the interface with GaAs as well as in the area adjacent to the surface, we performed calculations for an uncovered $(\mathrm{Ga}, \mathrm{Mn}) \mathrm{As}$ film as well as one with three additional capping layers of $\mathrm{Au}$. Due to the small amount of free charge carriers in $(\mathrm{Ga}, \mathrm{Mn}) \mathrm{As}$, the surface potential decays slowly into bulk, leading to a potential and a charge-density gradient within an extended region adjacent to the surface. The existence of such a potential gradient results in the breaking of the fourfold symmetry of the bulk (Ga,Mn)As system, making the [1ํㅣㄹ and [110] directions inequivalent (for the geometry used here this corresponds to the $x$ and $y$ directions, respectively) and leading effectively to a $C_{2 v}$ symmetry not only within the few surface and interface layers, but also in a rather extended subsurface regime.

We discuss now the surface-induced MCA in the film. Here we focus mainly on the MAE properties of a (Ga,Mn)As film with a clean Ga-terminated surface deposited on $\mathrm{GaAs}(001)$. The results for the energy differences between different magnetization directions are $E_{[110]}-E_{[001]}=-80.56 \mu \mathrm{eV}$ and $E_{[1 \overline{1} 0]}-E_{[001]}=-32.96 \mu \mathrm{eV}$ per film unit cell $(8 \mathrm{ML})$. This gives an uniaxial in-plane anisotropy with the energy difference of $E_{[110]}-E_{[1 \overline{1} 0]}=-47.6 \mu \mathrm{eV}$ per film unit cell.

Figure 2(a) presents the layer-resolved contributions to the $E_{[110]}-E_{[001]}$ and $E_{[1 \overline{1} 0]}-E_{[001]}$ values, indicated by open and filled symbols, respectively. The difference between these values characterizes the MCA within the plane. One should emphasize here that the contribution to the MCA from the region close to the surface decays slowly into the bulk. Therefore, the surface-induced anisotropy effect in the uniaxial in-plane MCA is determined by a region adjacent to the surface, extended rather deep into bulk, but not just by two or three subsurface layers, as it is often observed in metallic systems (e.g., Ref. 34). The corresponding contribution to the
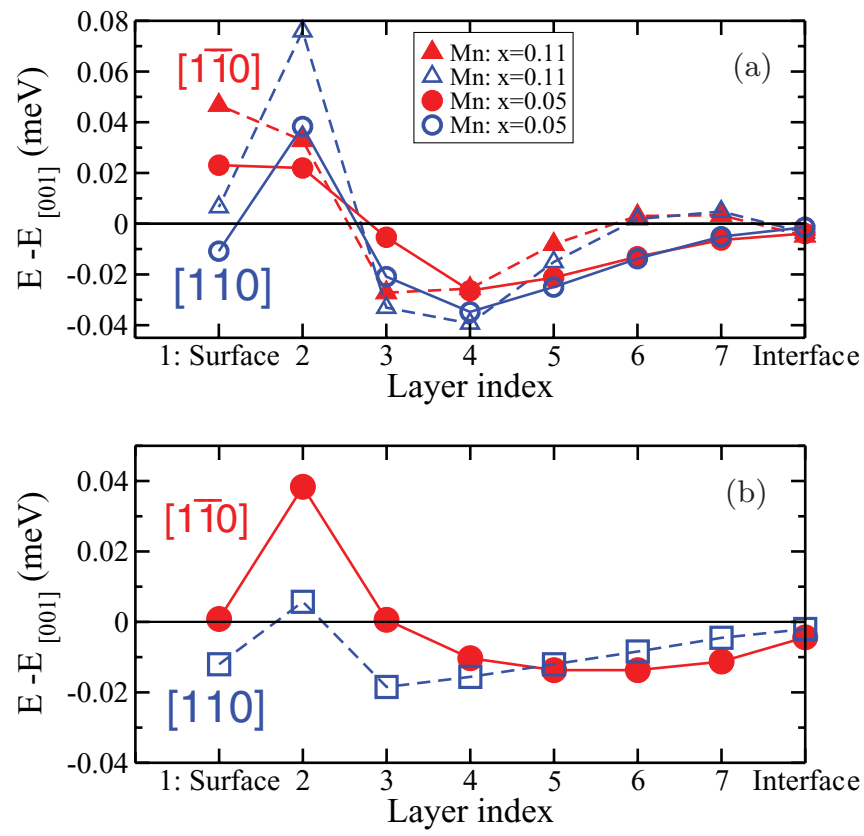

FIG. 2. (Color online) (a) Layer-resolved contributions to the MCA energy in the uncovered 8-ML (Ga,Mn)As film with 5 at. \% $\mathrm{Mn}$ (circles) and 11 at.\% Mn (triangles), for two directions of magnetization: $\vec{M} \|[110]$ and $\vec{M} \|[1 \overline{1} 0]$; (b) layer-resolved contributions to the MCA energy in the 8-ML (Ga,Mn)As film with 5 at. $\% \mathrm{Mn}$, with three capping layers of $\mathrm{Au}$.

energy of the uniaxial in-plane anisotropy exceeds by far the energy of the biaxial in-plane anisotropy when normalized to the same volume. Using these results, the MCA of experimental (Ga,Mn)As films consisting of $n+8$ monolayers can be modeled by combining the contribution of $n$ bulklike layers with the contribution of eight layers of surface region. This gives two competing contributions to the MCA: a biaxial in-plane anisotropy from bulklike layers of $(\mathrm{Ga}, \mathrm{Mn}) \mathrm{As}$ with a tetragonal distortion and a uniaxial in-plane anisotropy from the area adjacent to the surface. Applying our obtained MAE values to a unit volume, one can get the MCA of the whole film including the surface region. Within our consideration, the coefficient of the in-plane biaxial anisotropy does not depend on the film thickness $L$, while the coefficient of the in-plane uniaxial anisotropy $K_{\|}$recalculated per unit volume should decrease with film thickness as $1 / L$. Thus, according to our numerical results, a rather strong uniaxial anisotropy should be observed in the case of very thin films, while the increase of the film thickness should lead to a competition of biaxial and uniaxial anisotropies beginning with a certain film thickness. The contribution from the "surface" region to the out-of-plane uniaxial anisotropy $E_{[1 \overline{1} 0]}-E_{[001]}$ decreases as well with the film thickness as $1 / L$. This results in a leading role of the in-plane anisotropy contribution caused by the tetragonal lattice distortion discussed above. Note that an increase of the $\mathrm{Mn}$ concentration results in an increase of the charge carriers in the film, which again results in better screening of the surface potential. This can be seen in Fig. 2(a), where the values $E_{[110]}-E_{[001]}$ and $E_{[1 \overline{1} 0]}-E_{[001]}$ are shown as a function of the distance from the surface for a $(\mathrm{Ga}, \mathrm{Mn}) \mathrm{As}$ film with $11 \% \mathrm{Mn}$. This increase in Mn concentration results in an in-plane MAE $E_{[110]}-E_{[1 \overline{1} 0]}=-20.8 \mu \mathrm{eV}$ per film 

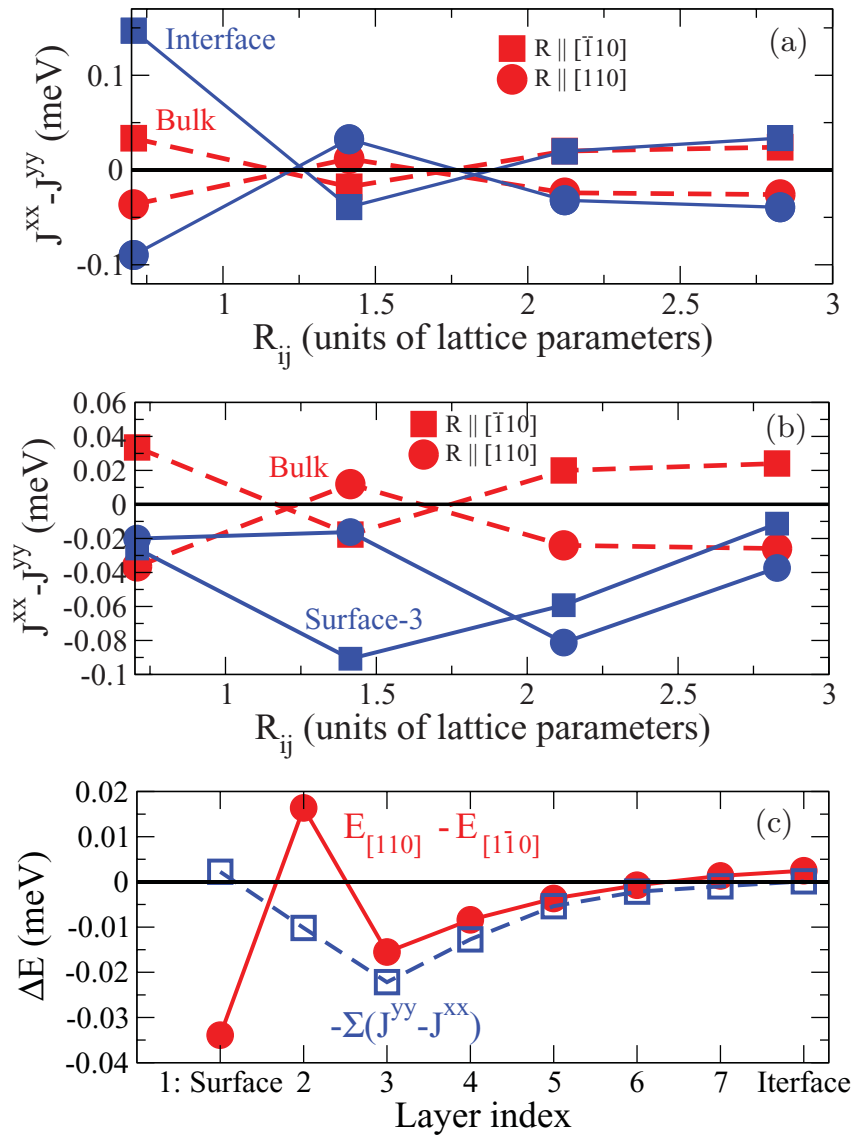

FIG. 3. (Color online) Variation of $\left(J_{i j}^{x x}-J_{i j}^{y y}\right)$ with distance $R_{i j}$ of pairs $(i, j)$ of Mn atoms taken in the [110] and [1 $1 \overline{10}]$ directions in the (Ga,Mn)As/GaAs film: (a) Bulk vs (Ga,Mn)As/GaAs interface and (b) bulk vs (Surf.-3) layer. (c) Layer-resolved sum $-\sum_{j}\left(J_{i j}^{y y}-J_{i j}^{x x}\right)$ calculated within the sphere of radius $2.9 a$ in comparison with the MCA energy $E^{[110]}-E^{[1 \overline{1} 0]}$ evaluated by magnetic torque calculations for the 8-ML (Ga,Mn)As film with 5 at.\% Mn.

unit cell, i.e., one obtains a smaller anisotropy energy when compared to the case of $5 \% \mathrm{Mn}$.

Since the uniaxial MCA has its origin in an extended subsurface region, one can expect that it is an intrinsic property of the systems and should be observed not only in the case of a clean surface but also in the presence of overlayers on the top of the (Ga,Mn)As film. Corresponding investigations have been performed for a $(\mathrm{Ga}, \mathrm{Mn})$ As film with three capping layers of $\mathrm{Au}$ on top of the (Ga,Mn)As film. The resulting layer-resolved contribution to the MCA is shown in Fig. 2(b). In spite of the differences in the MAE between the Au-capped (Ga,Mn)As film and the case of uncovered film, the general trend in both cases is the same, i.e., one can clearly see that the difference in layer contributions to the MCA for different directions of

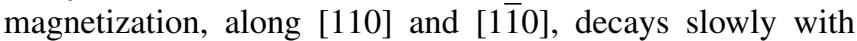
the distance from the surface or $\mathrm{Au} /(\mathrm{Ga}, \mathrm{Mn}) \mathrm{As}$ interface, respectively. Calculations for As-terminated surfaces and interfaces show similar layer-resolved MCA behaviors for magnetization along the [110] and [110] directions, as was obtained for Ga-terminated (Ga,Mn)As films.

To investigate also the effect caused by a concentration gradient along the surface normal within an uncovered (Ga,Mn)As film, we dealt with a corresponding film where the $\mathrm{Mn}$ concentration varies from $5 \%$ at the (Ga,Mn)As/GaAs interface to $6.6 \%$ in the surface layer. The calculations show that this does not result in a noteworthy change in the MCA.

To analyze in more detail the origin of the surface-induced in-plane uniaxial anisotropy, the contribution of the exchange interaction anisotropy in the (Ga,Mn)As film was determined. Figure 3 shows the difference $J_{i j}^{x x}-J_{i j}^{y y}$ calculated along the [110] and [1 $\overline{1} 0]$ directions within the film layers, where the $x$

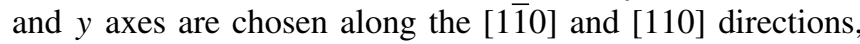
respectively. As discussed above, for bulk (Ga,Mn)As the variation of $J_{i j}^{x x}-J_{i j}^{y y}$ with distance $\left|\vec{R}_{i j}\right|$ is the same for $\vec{R}_{i j}$ along the [110] and [1 $\overline{1} 0]$, however, with a different sign. This behavior is more or less the same for Mn atoms next to the (Ga,Mn)As/GaAs interface [see Fig. 3(a)]. For Mn in the fourth layer (choosing the surface layer as the first layer), however, the situation is changed, indicating a pronounced modification of the anisotropic exchange coupling due to the broken symmetry. As a result, the sum over lattice sites in the expression for the effective anisotropy coefficients does not vanish, which leads to a contribution to the in-plane uniaxial anisotropy. Figure 3(b) shows the corresponding results obtained by summing the terms $\left(J_{i j}^{x x}-J_{i j}^{y y}\right)$ over all lattice sites $j$ within a sphere of radius $2.9 a$, with $i$ taken within the layers $1-8$ in the $(\mathrm{Ga}, \mathrm{Mn}) \mathrm{As}$ film. As one can see, the anisotropy of the exchange interaction indeed gives a substantial contribution to the anisotropy energy $E_{[110]}-E_{[1 \overline{1} 0]}$ for layers 3-8. For the first two film layers. i.e., surface and subsurface layers, the two curves strongly deviate, reflecting the dominating on-site contribution to the MCA. ${ }^{28,30}$

In summary, our results show that the tetragonal distortion [caused by a compressive strain due to lattice mismatch of (Ga,Mn)As and GaAs lattices] is responsible for the biaxial in-plane anisotropy that is in line with the interpretation given in previous investigations. A strong uniaxial in-plane MCA was found in the (Ga,Mn)As film in the area adjacent to the surface or an interface. We conclude that this is a result of the slow decay of the surface potential gradient due to the small amount of free charge carriers. The contribution to the uniaxial in-plane anisotropy decays rather slowly into the bulk and is not restricted to only a few surface layers. Moreover, a significant contribution responsible for the MCA in the films is caused by the anisotropic Mn-Mn exchange interactions mediated by holes in the valence band of $(\mathrm{Ga}, \mathrm{Mn}) \mathrm{As}$.

Financial support by the DFG through the SFB 689 is gratefully acknowledged.
${ }^{1}$ T. Jungwirth, J. Sinova, J. Mašek, J. Kučera, and A. H. MacDonald, Rev. Mod. Phys. 78, 809 (2006).

${ }^{2}$ T. Dietl, H. Ohno, and F. Matsukura, Phys. Rev. B 63, 195205 (2001).
${ }^{3}$ M. Abolfath, T. Jungwirth, J. Brum, and A. H. MacDonald, Phys. Rev. B 63, 054418 (2001).

${ }^{4}$ L. M. Sandratskii and P. Bruno, in Local-Moment Ferromagnets: Unique Properties for Modern Applications, edited by M. Donath 
and W. Nolting, Lecture Notes in Physics Vol. 678 (Springer, Berlin, 2005), p. 113.

${ }^{5}$ C. Sliwa and T. Dietl, Phys. Rev. B 78, 165205 (2008)

${ }^{6}$ T. O. Strandberg, C. M. Canali, and A. H. MacDonald, Phys. Rev. B 81, 054401 (2010).

${ }^{7}$ M. Sawicki, Acta Physica Polonica A 106, 119 (2004).

${ }^{8}$ T. Dietl, Physica E 10, 120 (2001).

${ }^{9}$ M. Sawicki, F. Matsukura, T. Dietl, G. M. Schott, C. Ruester, G. Schmidt, L. W. Molenkamp, and G. Karczewski, J. Supercond. 16, 7 (2003).

${ }^{10}$ X. Liu, Y. Sasaki, and J. K. Furdyna, Phys. Rev. B 67, 205204 (2003).

${ }^{11}$ K. Hamaya, T. Watanabe, T. Taniyama, A. Oiwa, Y. Kitamoto, and Y. Yamazaki, Phys. Rev. B 74, 045201 (2006).

${ }^{12}$ D. Y. Shin, S. J. Chung, S. Lee, X. Liu, and J. K. Furdyna, Phys. Rev. B 76, 035327 (2007).

${ }^{13}$ U. Welp, V. K. Vlasko-Vlasov, X. Liu, J. K. Furdyna, and T. Wojtowicz, Phys. Rev. Lett. 90, 167206 (2003).

${ }^{14}$ H. X. Tang, R. K. Kawakami, D. D. Awschalom, and M. L. Roukes, Phys. Rev. Lett. 90, 107201 (2003).

${ }^{15}$ K.-Y. Wang, M. Sawicki, K. W. Edmonds, R. P. Campion, S. Maat, C. T. Foxon, B. L. Gallagher, and T. Dietl, Phys. Rev. Lett. 95, 217204 (2005).

${ }^{16}$ A. Sugawara, H. Kasai, A. Tonomura, P. D. Brown, R. P. Campion, K. W. Edmonds, B. L. Gallagher, J. Zemen, and T. Jungwirth, Phys. Rev. Lett. 100, 047202 (2008).

${ }^{17}$ D. Hrabovsky, E. Vanelle, A. R. Fert, D. S. Yee, J. P. Redoules, J. Sadowski, J. Kanski, and L. Ilver, Appl. Phys. Lett. 81, 2806 (2002).

${ }^{18}$ M. Sawicki, K.-Y. Wang, K. W. Edmonds, R. P. Campion, C. R. Staddon, N. R. S. Farley, C. T. Foxon, E. Papis, E. Kaminska, A. Piotrowska, T. Dietl, and B. L. Gallagher, Phys. Rev. B 71, 121302(R) (2005).

${ }^{19}$ M. Glunk, J. Daeubler, L. Dreher, S. Schwaiger, W. Schoch, R. Sauer, W. Limmer, A. Brandlmaier, S. T. B. Goennenwein, C. Bihler, and M. S. Brandt, Phys. Rev. B 79, 195206 (2009).
${ }^{20}$ A. W. Rushforth, E. De Ranieri, J. Zemen, J. Wunderlich, K. W. Edmonds, C. S. King1, E. Ahmad, R. P. Campion, C. T. Foxon, B. L. Gallagher, K. Výborný, J. Kučera, and T. Jungwirth, Phys. Rev. B 78, 085314 (2008).

${ }^{21}$ J. Zemen, J. Kučera, K. Olejník, and T. Jungwirth, Phys. Rev. B 80, 155203 (2009).

${ }^{22}$ A. Werpachowska and T. Dietl, Phys. Rev. B 82, 085204 (2010).

${ }^{23}$ M. Kopecký, J. Kub, F. Máca, J. Mašeck, O. Pacherová, A. W. Rushforth, B. L. Gallagher, R. P. Campion, V. Novák, and T. Jungwirth, Phys. Rev. B 83, 235324 (2011).

${ }^{24}$ H. Ebert, in Electronic Structure and Physical Properties of Solids, edited by H. Dreyssé, Lecture Notes in Physics Vol. 535 (Springer, Berlin, 2000), p. 191.

${ }^{25} \mathrm{H}$. Ebert et al., The Munich SPR-KKR package, version 5.4 [http://olymp.cup.uni-muenchen.de/ak/ebert/SPRKKR] (2009).

${ }^{26}$ J. B. Staunton, L. Szunyogh, A. Buruzs, B. L. Gyorffy, S. Ostanin, and L. Udvardi, Phys. Rev. B 74, 144411 (2006).

${ }^{27}$ X. Wang, D. Wang, R. Wu, and A. J. Freeman, J. Magn. Magn. Mater. 159, 337 (1996).

${ }^{28}$ L. Udvardi, L. Szunyogh, K. Palotás, and P. Weinberger, Phys. Rev. B 68, 104436 (2003).

${ }^{29}$ H. Ebert and S. Mankovsky, Phys. Rev. B 79, 045209 (2009).

${ }^{30}$ S. Mankovsky, S. Bornemann, J. Minár, S. Polesya, H. Ebert, J. B. Staunton, and A. I. Lichtenstein, Phys. Rev. B 80, 014422 (2009).

${ }^{31}$ K. Y. Wang, A. W. Rushforth, V. A. Grant, R. P. Campion, K. W. Edmonds, C. R. Staddon, C. T. Foxon, B. L. Gallagher, J. Wunderlich, and D. A. Williams, J. Appl. Phys. 101, 106101 (2007).

${ }^{32}$ J. Daeubler, S. Schwaiger, M. Glunk, M. Tabor, W. Schoch, R. Sauer, and W. Limmer, Physica E 40, 1876 (2008).

${ }^{33}$ M. Cubukcu, H. J. von Bardeleben, K. Khazen, J. L. Cantin, O. Mauguin, L. Largeau, and A. Lemaitre, Phys. Rev. B 81, 041202 (2010).

${ }^{34}$ M. Košuth, Ph.D. thesis, University of Munich, 2007. 